\title{
Esophageal Leiomyoma Mimicking As Duplication Cyst
}

\author{
Authors \\ Girish D. Bakhshi, Rajesh G. Chincholkar, Prakash B. Patodekar, Chaitanaya G. Patil, \\ Rajesh B. Yadav, Amarjeet E. Tandur \\ Department of General Surgery, Grant Government Medical College \& Sir J.J. Group Of Hospitals, \\ Mumbai-08 INDIA
}

\begin{abstract}
Esophageal duplication cyst is a rare entity but well documented. Majority of them are usually diagnosed in childhood but adults are more likely to be symptomatic. Endoscopic ultrasound (EUS) is a diagnostic tool of choice to investigate duplication cyst since it can differentiate between solid and cystic component. However, esophageal duplication cyst, due to its solid content can masquerade as leiomyoma or other benign lesions, but it is exceptionally rare where leiomyoma of esophagus presented as homogenous anechoic lesion mimicking a duplication cyst. We present a case which posed a diagnostic dilemma with EUS appearances closely resembling esophageal duplication cyst, whereas CT scan was suggestive of leiomyoma. However intraoperative findings and histopathological examination of specimen confirmed it to be esophageal leiomyoma.
\end{abstract}

\section{INTRODUCTION}

Esophageal leiomyomas, though very rare, are most common benign tumours of esophagus ${ }^{[1]}$. These represent a hyper proliferation of interlacing bundles of smooth muscle cell well demarcated by surrounding tissue or connective tissue capsule ${ }^{[2]}$. Growing slowly esophageal leiomyomas are usually asymptomatic. most common presenting symptom being dysphagia. Large tumours can cause vague retrosternal discomfort, chest pain, esophageal obstruction, regurgitation and very rarely gastrointestinal bleeding ${ }^{[3]}$. Diagnosis is with help of esophagogram, esophagoscopy, computerized tomography (CT) scan, endoscopic ultrasound (EUS) ${ }^{[4]}$. Enucleation of leiomyoma is safe and effective procedure but whether to operate every patient of leiomyoma remains a controversy. We present a case with diagnostic dilemma as the esophageal lesion was diagnosed as duplication cyst on EUS and leiomyoma on CT scan.

\section{CASE HISTORY}

A 24-years-old male presented with complaints of heartburn and progressive dysphagia to solids and liquids since 3 months. Patient had previous CT report suggestive of eccentric of dimensions $6.4 \times 3.2 \times 6.4 \mathrm{~cm}$ with central ulceration communicating with lumen. A few calcific foci within. Focal loss of fat plane with aorta. No lymphadenopathy. GIST being the main differential diagnosis (Fig. 1). 


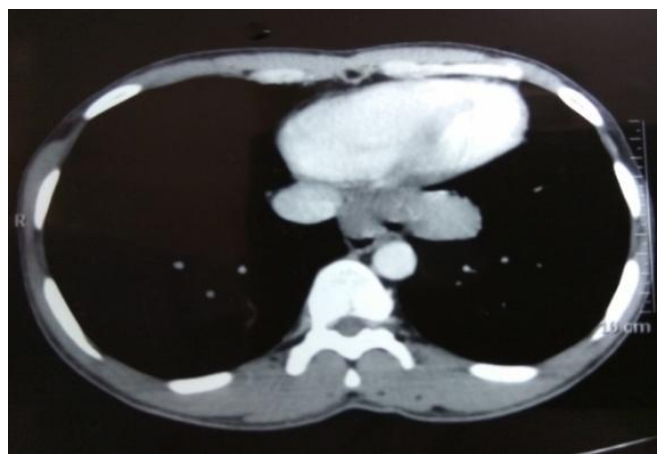

Fig. 1- CT image showing mildly enhancing mural mass lesion in lower esophagus.

Patient was non diabetic, non-hypertensive, had no other co-morbidities. There was no previous history of any trauma or surgery. Patient also denied history of any addiction or drug abuse. Both General \& Systemic examination was unremarkable.. All routine blood investigation reports were within normal limits. ECG and chest \& abdominal X ray were grossly normal.

Esophagogastroscopy revealed bulge in lumen of esophagus at $35 \mathrm{~cm}$ from incisors suggesting extrinsic compression along with transverse elongation of the lumen (Fig. 2). Patient's endoscopic ultrasound was done which suggested large anechoic mass of size $7 \times 4 \mathrm{~cm}$ in lower esophagus with echogenic parts in some parts which appears like a large duplication cyst rather than GIST (Fig. 3). Repeat CT scan done at our centre suggested a non-enhancing circumferential thickening involving distal esophagus with few foci of calcification in lesion with proximal dilation of esophagus (Fig. 4). In CT scan lesion appeared most likely to be leiomyoma and differentials being GIST communicating with esophagus and lymphoma.

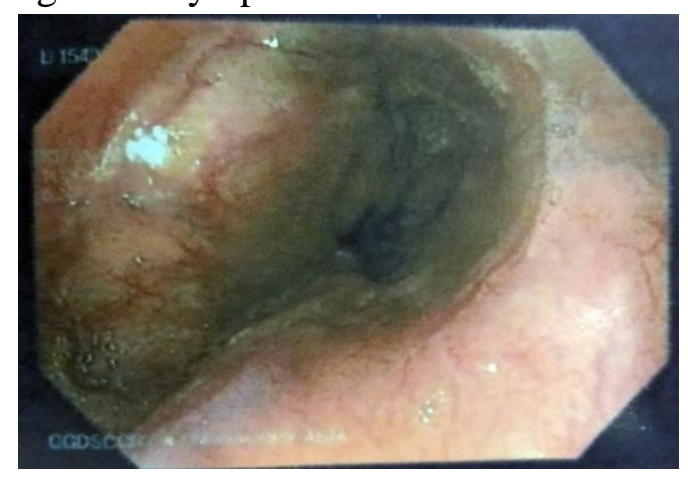

Fig. 2- Esophagoscopy showing extrinsic compression at $35 \mathrm{~cm}$ from incisors with intact mucosa

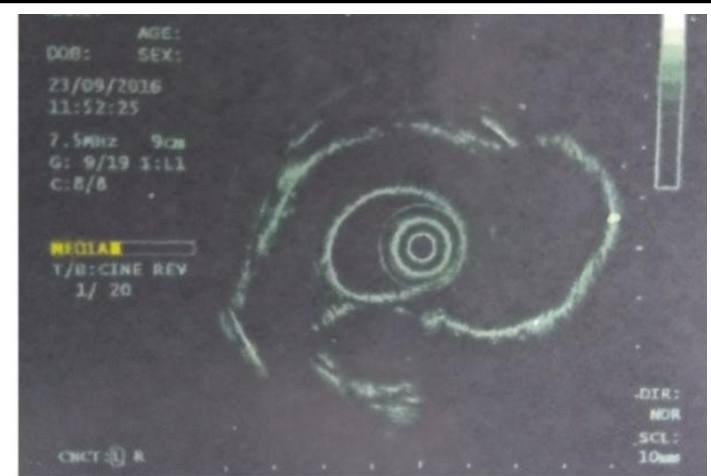

Fig. 3- Endoscopic ultrasound showing large anechoic mass of size $7 \times 4 \mathrm{~cm}$.

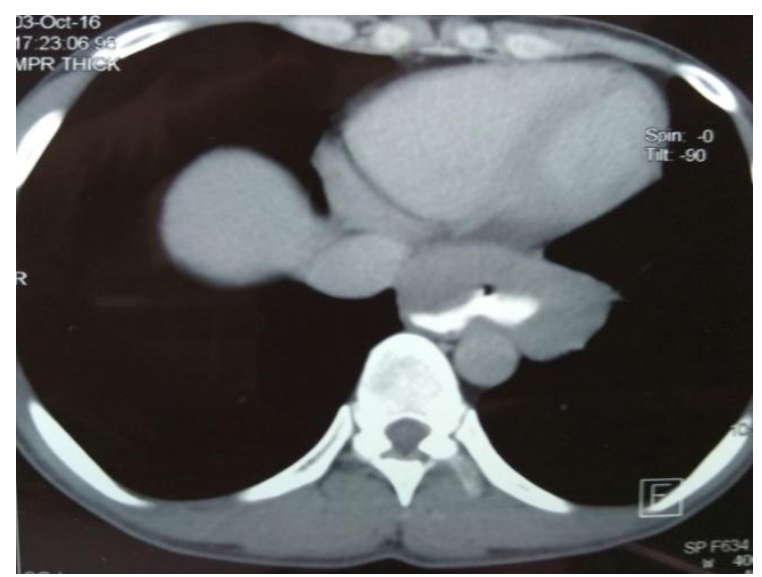

Fig. 4- Repeat CT scan showing large non enhancing circumferential thickening in distal third of esophagus.

After obtaining anesthesia fitness and required consent, patient underwent laparotomy. Gastroesophageal (GE) junction was mobilized. Hiatus was widened to gain access to lower end of esophagus. Intraoperatively a large elongated firm intramuscular well defined tumour was noted in lower part of esophagus with well-preserved planes and intact mucosa which was not consistent with duplication cyst (Fig. 5). Enucleation of tumour was done. There was no evidence of mucosal breach as confirmed with intraoperative esophagoscopy and esophageal leak test (Fig. 6). Muscular layer was approximated with intermittent sutures. Hiatus was repaired and Dor's anterior fundoplication was done. Tumour was sent for histopathological examination which confirmed it to be leiomyoma. Histopathologically sections revealed circumscribed lobulated spindle cell tumour with tumour cells arranged in interlacing fascicles and showing abundant 
eosinophilic cytoplasm. No evidence of mitotic activity seen. Furthermore, on immunohistochemistry, the tumour cell were positive for SMA and Desmin while being negative for S100, DOG1 and Ckit (ruling out gastro intestinal stromal tumours).

Patient's Post-operative period was uneventful and was discharged on day 12 after surgery. Follow up of 6 months has shown him to be symptom and disease free.

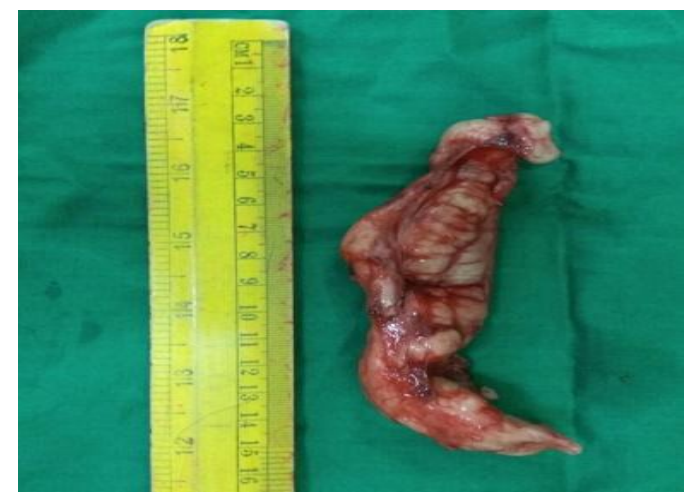

Fig. 5 - Enucleated surgical specimen

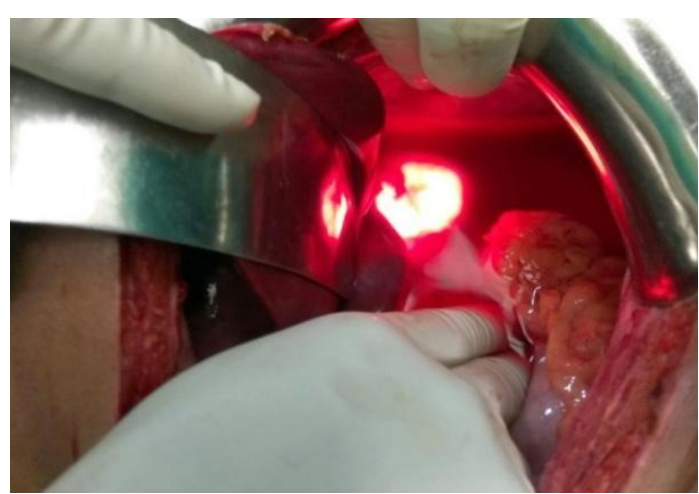

Fig. 6- Intra operative esophagoscopy and mucosal leak test being performed after enucleation of tumour to check integrity of mucosa.

\section{DISCUSSION}

Esophageal Leiomyoma is most common benign esophageal tumour accounting for two third of all esophageal benign tumours ${ }^{[5]}$. It occurs twice as common in males than females. About $60 \%$ of these tumours are located in distal third of esophagus ${ }^{[5]}$ and are multiple in approximately $5 \%$ of patients ${ }^{[3,5]}$.

About half of the patients with leiomyoma are asymptomatic; when symptoms do occur, they are fairly long standing and include retrosternal pain and dysphagia. In large tumours patient may also present with esophageal obstruction, regurgitation, weight loss and muscle wasting ${ }^{[5]}$. Also quite rarely bleeding may occur when mucosa over tumour ulcerate ${ }^{[1]}$. On one hand, few authors consider that there is no direct relation between size of tumour and symptoms ${ }^{[6]}$, while others have correlated that a tumour size of more than $5.3 \mathrm{~cm}$ can cause symptoms ${ }^{[3]}$. In present case patient presented with dysphagia and heartburn.

Radiologically leiomyoma may appear as mediastinal mass on plain chest $\mathrm{X}$ ray and luminal filling defect on barium swallow [4] Esophagoscopy may be normal or show extrinsic compression; however mucosal ulceration may also be evident rarely in which case biopsy is indicated to rule out malignancy ${ }^{[6]}$. Computerized Tomography (CT) elaborates anatomical relationship of tumour and differentiate intramural lesions from extrinsic ones; however, differentiating from other soft tissue lesion like fibroma, neurofibroma and hemangioma may be difficult ${ }^{[7]}$. Leiomyoma appears as homogenous mass on CT. T2-weighted MR imaging shows a slightly hyper intenselesion ${ }^{[8]}$.

Recently, Endoscopic ultrasonography (EUS) has gained popularity as it can differentiate esophageal cystic and soft tissue lesions accurately, also it can demonstrate exact location of mass in relation to esophageal wall and mediastinum. Leiomyoma, on EUS, is seen as homogenous regions of hypoechogenecity juxtaposed with overlying mucosa ${ }^{[9]}$. In present case, EUS revealed large anechoic mass with few echogenic content mimicking as duplication cyst, and also these findings were extremely unusual for leiomyoma. In fact, only one case has been reported in literature where esophageal lesion, on EUS, have been seen as anechoic mass mimicking cyst but confirmed to be leiomyoma on histopathology ${ }^{[10]}$.

Histologically, leiomyoma comprises of bundles of interlacing smooth muscle cells and fascicle of spindle cell, well demarcated by adjacent tissue or by definitive connective tissue capsule. Tumour 
cell may show few or no mitotic activity ${ }^{[2]}$. Furthermore, leiomyoma may undergo cystic degeneration, calcification and infrequently, malignant transformation [3]. In present case histopathological study revealed circumscribed lobulated spindle cell tumour with tumour cells arranged in interlacing fascicles and showing abundant eosinophilic cytoplasm and with no evidence of mitotic activity.

Literature recommend resection of all leiomyoma which are symptomatic and also for asymptomatic when size is more than $5 \mathrm{~cm}$, continued increase in size or with mucosal breach or ulceration ${ }^{[11]}$. Few authors have opted for non-surgical management and endoscopic follow up in asymptomatic and moderately symptomatic cases ${ }^{[12]}$. In present case leiomyoma was more than 5 $\mathrm{cm}$ and patient was symptomatic hence excision was planned.

Tumour removal can be done either by thoracotomy with esophageal resection or enucleation of tumour by thoracoscopic approach. Tumours at gastroesophageal junction can be addressed through upper midline laparotomy as in present case. Esophageal resection is recommended for tumour of size more than $8 \mathrm{~cm}$, when tumour is adherent to mucosa or if there is extensive mucosal damage during dissection ${ }^{[2,6]}$. However, authors have performed enucleations of tumours more than $8 \mathrm{~cm}$ size ${ }^{[13]}$. Some authors prefer not to approximate muscle layer after myotomy provided mucosal layer is intact ${ }^{[2]}$ while many others recommend approximating myotomy defect for prevention of diverticula and also postoperative reflux esophagitis ${ }^{[14]}$.

\section{CONCLUSION}

Although newer imaging modalities may help in clinching diagnosis of various esophageal tumours accurately, sometimes absence of characteristic findings may create a dilemma. In all cases presenting with esophageal symptoms, one must not rely completely on imaging and be suspicious as leiomyoma may mimic as duplication cyst and vice versa.

\section{REFERENCES}

1. Zhang $\mathrm{W}$, Xue $\mathrm{X}$, Zhou Q. Benign esophageal schwannoma. South Med J, 2008; 101: 450-1.

2. Evans HL. Smooth muscle tumours of gastrointestinal tract: A study of 56 cases followed for a minimum of 10 years. Cancer 1985; 56:2242-50.

3. Mutrie CJ, Donahue DM, Wain JC, Wright $\mathrm{CD}$, Gaissert HA, et al. Esophageal leiomyoma: a 40-year experience. Ann Thorac Surg. 2005; 79: 1122-5.

4. Yang PS, Lee KS, Lee SJ, et al. Esophageal leiomyoma: radiologic findings in 12 patients. Korean J Radiol 2001;2:132-7.

5. Seremetis MG, Lyons WS, deGuzman VC, Peabody JW JrLeiomyomata of the esophagus. An analysis of 838 cases. 1976; 38: 2166-77.

6. Wang Y, Zhang R, Ouyang Z, Zhang D, Wang L, Zhang D. Diagnosis and surgical treatment of esophageal leiomyoma. Zhonghua Zhong Liu Za Zhi 2002; 24: 394-6.

7. Karagülle E, Akkaya D, Türk E, Göktürk HS, Yildirim E, et al. Giant leiomyoma of the esophagus: a case report and review of the literature. Turk J Gastroenterol 2008; 19: 180-3.

8. Po SY, Kyung SL, Soon JL, Tae SK, InWook C, Young MS, Kwhanmien K, Yookyung K. Esophageal leiomyoma: radiologic findings in 12 patients. Korean $\mathbf{J}$ Radiol 2001;2: 132-7.

9. Massari M, De Simone M, Cioffi U, Gabrielli F, Boccasanta P, Bonavina L. Endoscopic ultrasonography in the evaluation of leiomyoma and extramucosal cysts of the esophagus. Hepatogastroenterology 1998; 45: 938-43.

10. Chien-Chu Lin, Jyh-Chin Yang, Hsiu-Po Wang, Jang-Ming Lee, Kao-Lang Liu. Annular Esophageal Leiomyoma Mimicking a Foregut Cyst on Endoscopic Ultrasound. J Med Ultrasound 2006;14:6-10. 
11. Priego P, Lobo E, Alonso N, Gil Olarte MA, Pérez de Oteyza J, Fresneda V. Surgical treatment of esophageal leiomyoma: an analysis of our experience. Rev Esp Enferm Dig 2006;98:350-8.

12. Glanz I, Grünebaum M. The radiological approach to leiomyoma of the oesophagus with a long-term follow-up. Clin Radiol 1977; 28: 197-200.

13. Kent $M$, d'Amato $T$, Nordman $C$, Schuchert M, Landreneau $\mathrm{R}$, et al.Minimally invasive resection of benign esophageal tumors. J Thorac Cardiovasc Surg 2007; 134: 176-81.

14. Lee LS, Singhal S, Brinster CJ, Marshall B, Kochman ML, et al. Current management of esophageal leiomyoma. J Am Coll Surg 2004; 198: 136-46. 